\title{
AN INSTRUCTIVE ALGORITHM FOR AIRCRAFT ELEVATOR SIZING TO BE USED IN PRELIMINARY AIRCRAFT DESIGN SOFTWARE
}

\author{
Omran Al-Shamma ${ }^{1^{*}}$ Rashid Ali ${ }^{2}$ Haitham S. Hasan ${ }^{1}$ \\ ${ }^{1}$ University of Information Technology and Communications, Baghdad, Iraq \\ ${ }^{2}$ Sheffield Hallam University, UK
}

The longitudinal control is the essential elevator function. For a safe flight, the aircraft should be longitudinally controllable plus maneuverable. So, it is imperative for the designers to assess the control authority of candidate configurations early in the preliminary design phase. Adding elevator sizing module, as a helpful tool for aeronautical students enhance their knowledge, understanding, and analyzing studies. This paper presents an instructive algorithm for the elevator sizing to be employed in the preliminary aircraft design software. The paper introduced the necessary formulae to guide the designer to achieve the qualifications of the longitudinal control and longitudinal trim. A solved example has been added to explain the application of the algorithm.

Key words: Algorithms, Elevators, Elevator sizing, Control surface design, Preliminary aircraft design

\section{INTRODUCTION}

The longitudinal control is the essential elevator function. For a safe flight, the aircraft should be longitudinally controllable plus maneuverable. So, it is imperative for the designers to assess the control authority of candidate configurations early in the preliminary design phase. This early assessment formulates the design process very proficient and cost-effective. Elevator sizing is one of the controllability concepts that should be involved in the preliminary design process. Adding elevator sizing module, as a helpful tool for aeronautical students enhance their knowledge, understanding, and analyzing studies.

The longitudinal control can be accomplished by affording an additional lift force on the horizontal tail [01]. It is basically directed through elevator deflection and engine throttle setting. In addition, it is ruled during the pitch rate, and as a result the angular acceleration, around the $y$-axis. Hence, the elevator is categorized as a pitch control device. Note that the longitudinal control of the conventional aircraft is not joined with the lateral-directional control, and therefore, the elevator sizing process is unrelated to rudder sizing process [02]. This matter makes elevator design simpler.

A quick pass on the available developed software for preliminary aircraft design is publicized briefly. Starting with Roskam's software (AAA) [03], it is basically a coded version of his textbook [04]. Raymer in 1996 released his software package (ADS) [05] based also on his book [06]. Soon later, the comprehensive software CEASIOM [07] was developed in 2008 and still in the stage of improvement. Finally, Nicolosi released his software (ADAS) [08] in 2011. Unfortunately, all these software packages present the elevator sizing indirectly by offering just the stability and controllability derivatives, dimensional and non-dimensional. These derivatives are not so clear for students, and many practices must be exercised to be familiar with. Typically, the elevator sizing process is done in the detail phase.

From the research side view, the current researches are investigating different aspects of the elevator other than elevator sizing, such as, elevator jam failures [09] [10], elevator actuation system [11], and elevator performance [12] [13]. All these researches are proposed for the aircraft industry and are not planned for instructional use., which consecutively, are out of the scope of the paper.

\section{ELEVATOR SIZING PRINCIPLES}

As mentioned above, the fundamental principle behind elevator is to change the horizontal tail pitching moment. Due to the elevator deflection up or down, the horizontal tail lift coefficient $\left(C_{L_{h}}\right)$ will decrease or increase, respectively. Hence, the two main objectives of the elevator are the longitudinal control and longitudinal trim.

In the elevator sizing process, three significant factors need to take into consideration which are: elevator aerodynamic and mass balancing, elevator effectiveness, and elevator hinge moment. The first factor deals with the method of varying the hinge moment in order to keep the stick force within an acceptable range and without the occurrence of aeroelastic trend. The second factor is affected by the tail moment arm and the elevator size. The importance of the last factor is to defeat the aerodynamic moment to rotate the elevator [14].

However, the prerequisites of longitudinal control handling quality during takeoff rotation of the takeoff stage are identified for a conventional tricycle aircraft as: there is a value for the pitch rate in case that the duration of the takeoff rotation must not arise over a predefined period of time. According to Newton's second law, the time of the takeoff rotation can be extracted in terms of the angular acceleration of the aircraft around the rotation 
point of the main gear [15]. For example, the tolerable time of the takeoff rotation of a transport aircraft is 3-5 seconds, and the corresponding angular rotation rate to satisfy that prerequisite is $4-6 \mathrm{deg} / \mathrm{s}^{2}$ where the center of aircraft gravity is positioned at the most forward location. Table 1 shows different types of aircraft with its qualifications of takeoff angular acceleration, which in turn, are extremely helpful in the elevator sizing process.

Table 1: The qualifications of takeoff angular acceleration

\begin{tabular}{|c|c|c|}
\hline Aircraft Type & $\begin{array}{c}\text { Takeoff Rotation } \\
\text { Time (s) }\end{array}$ & $\begin{array}{c}\text { Takeoff Pitch An- } \\
\text { gular Acceleration } \\
\left(\text { deg/s }{ }^{2}\right.\end{array}$ \\
\hline Large transport & $3-5$ & $4-6$ \\
\hline Small transport & $2-4$ & $6-8$ \\
\hline $\begin{array}{c}\text { Normal general } \\
\text { aviation }\end{array}$ & $1-3$ & $8-10$ \\
\hline $\begin{array}{c}\text { Utility, } \\
\text { semi-acrobatic } \\
\text { GA }\end{array}$ & $1-2$ & $10-15$ \\
\hline $\begin{array}{c}\text { Highly maneu- } \\
\text { verable (e.g., } \\
\text { fighter) }\end{array}$ & $0.2-0.7$ & $12-20$ \\
\hline
\end{tabular}

Prior to elevator sizing, three ratios relating the elevator to horizontal tail and one parameter must be assigned which are: $S_{e} / S_{h}, b_{e} / b_{h}, C_{e} / C_{h}$, and $\pm \delta_{e}$ max. The typical values [16] are: $0.15,-0.4,0.8,-1.0,0.26,-0.34, \pm$ $25 \mathrm{deg}$, respectively. Due to effortlessness in sizing and manufacturing, the elevator span is frequently chosen to be the same as the span of the horizontal tail, i.e., $b_{\mathrm{e}} / b_{h}=1$. Also, to avoid flow separation which leads the horizontal tail to stall, the maximum elevator deflection (up or down) should not exceed 25 degrees. With elevator of full span and maximum allowable deflection, the chord of the elevator should be enlarged to produce the required change in the tail lift. An all-moving tail is a choice, if the requisite elevator chord becomes greater than the half of the chord of the horizontal tail as in fighter aircraft to produce enough pitching moment for highly maneuverable.

The analysis of a tricycle conventional aircraft equilibrium at rotation point can be accomplished by the following three equations, two of them are force equations (Equations 1 and 2) and the third is a moment equation (Equation 3). Note that the moments with clockwise direction are positive while the counterclockwise are negative. Therefore, weight, thrust, and wing/fuselage pitching moments are negative as in Equation 3:

$$
T-D-F_{f}=m a
$$

where:

$$
D_{\text {to }}=\frac{1}{2} \rho v_{r}^{2} C_{d_{t o}} S_{r e f}
$$

$$
\begin{aligned}
& C_{d_{\text {to }}}=C_{d_{0}}+K C_{l_{\text {to }}}^{2} \\
& C_{l_{\text {to }}}=C_{l_{c}}+C_{l_{\text {flap }}} \\
& C_{l_{c}}=2 W /\left(\rho v_{c}^{2} S\right) \\
& F_{f}=\mu N \\
& N=W-L_{\text {to }}
\end{aligned}
$$

where:

$$
\begin{aligned}
& L_{t 0}=L_{w f}+L_{h} \\
& L_{w f} \cong \frac{1}{2} \rho v_{r}^{2} C_{L_{t o}} S_{r e f} \\
& L_{h}=\frac{1}{2} \rho v_{r}^{2} C_{L_{h}} S_{h} \\
& -M_{W}+M_{D}-M_{T}+M_{L_{w f}}+ \\
& +M_{a c_{w f}}+M_{L_{h}}+M_{a}=I_{y y_{m g}} \ddot{\theta}
\end{aligned}
$$

where:

$$
\begin{aligned}
& M_{W}=W\left(x_{m g}-x_{c g}\right) \\
& M_{D}=D\left(z_{D}-z_{m g}\right) \\
& M_{T}=T\left(z_{T}-z_{m g}\right) \\
& M_{L_{w f}}=L_{w f}\left(x_{m g}-x_{a c_{w f}}\right) \\
& M_{a c_{w f}}=\frac{1}{2} \rho v_{r}^{2} C_{m_{a c_{w f}}} S_{r e f} \bar{C} \\
& M_{L_{h}}=L_{h}\left(x_{a c_{h}}-x_{c g}\right) \\
& M_{a}=m a\left(z_{c g}-z_{m g}\right)
\end{aligned}
$$

Substituting Equations $3 \mathrm{a}$ to $3 \mathrm{~g}$ into Equation 3, and reformatting it for $L_{h}$, yields:

$$
L_{h}=\frac{\left(\begin{array}{c}
L_{w f}\left(x_{m g}-x_{a c_{w f}}\right)+M_{a c_{w f}}+m a\left(z_{c g}-z_{m g}\right)- \\
-W\left(x_{m g}-x_{c g}\right)+D\left(z_{D}-z_{m g}\right)-T\left(z_{T}-z_{m g}\right)-I_{y y_{m g}} \ddot{\theta}
\end{array}\right)}{x_{a c_{h}}-x_{m g}}
$$

The contribution of the elevator to the horizontal tail lift can be expressed with respect to tail lift coefficient as follows:

$$
C_{L_{h}}=\frac{2 L_{h}}{\rho v_{r}^{2} S_{h}}
$$

In general, this coefficient is negative and it affected by many factors related to the horizontal tail such as the features of the airfoil section [17], the angle of attack $(\alpha h)$, sweep angle, aspect ratio and taper ratio. Hence, this coefficient can be adapted as:

$$
C_{L_{h}}=C_{L_{\propto_{h}}} \propto_{h}+C_{L_{\propto_{h}}} \tau_{e} \delta_{e}
$$


where:

$$
\begin{aligned}
& \alpha_{h}=\alpha+i_{h}-\varepsilon \\
& \varepsilon=\varepsilon_{0}+\frac{\partial \varepsilon}{\partial \alpha} \alpha_{w}
\end{aligned}
$$

The elevator designer can influence the amount of the elevator control power through a suitable assortment of the elevator geometry. By employing Equation 6, one can establish the attributes of the elevator that achieve the takeoff rotation prerequisite. For instance, deliberating elevator angle of attack effectiveness, the designer can evaluate the elevator cord-to-tail ratio by using Figure 1 [18], and consecutively, the minimum elevator area that agrees with the majority critical aircraft longitudinal control prerequisite.

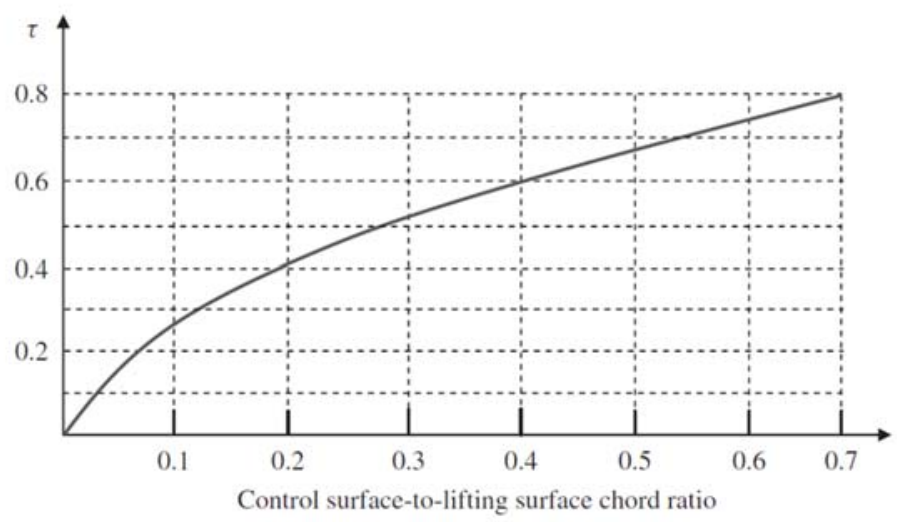

Figure 1: A general representation of the control surface effectiveness [18]

The following equation represents the mathematical model of Figure 1 using MATLAB, curve fitting tool [19]:

$$
\begin{aligned}
\tau_{a}= & -6.624\left(\frac{S_{a}}{S}\right)^{4}+12.07\left(\frac{S_{a}}{S}\right)^{3}- \\
& -8.292\left(\frac{S_{a}}{S}\right)^{2}+3.295\left(\frac{S_{a}}{S}\right)+0.004942
\end{aligned}
$$

On the trim side, the aircraft is to be in longitudinal trim if and only if all longitudinal forces and moments are in equilibrium. The following equation obtained the elevator deflection, which must be big enough to keep the aircraft longitudinal trim in all flight conditions:

$$
\delta_{e}=\frac{\left(\frac{T * Z_{T}}{\bar{q} * S * \bar{C}}+C_{m_{0}}\right) C_{L_{\alpha}}+\left(C_{L_{l}}-C_{L_{0}}\right) C_{m_{\alpha}}}{C_{L_{\alpha}} C_{m_{\delta_{e}}}-C_{m_{\alpha}} C_{L_{\delta_{e}}}}
$$

where:

$$
\begin{aligned}
C_{m_{\delta_{e}}} & =-C_{L_{\alpha_{h}}} \eta_{h} \bar{V}_{h} \frac{b_{e}}{b_{h}} \tau_{e} \\
C_{L_{\delta_{e}}} & =C_{L_{\alpha_{h}}} \eta_{h} \frac{s_{h}}{S} \frac{b_{e}}{b_{h}} \tau_{e}
\end{aligned}
$$

It should be noted that $z_{T}$ is negative if the thrust line is higher than the aircraft cg. Also, if the qualification of the elevator angle is more than $30 \mathrm{deg}$, one needs to enlarge the elevator size and/or the tail arm. Furthermore, the designer must take into consideration that the elevator deflection will decrease the tail stall angle and may cause the horizontal tail to stall. Therefore, it is recommended to keep the tail in the range of two degrees of its stall angle.

The tail angle of attack is defined as:

$$
\alpha_{h}=\alpha+i_{h}-\varepsilon
$$

Note that $\alpha$ at the onset of rotation (i.e., on the ground) is typically equal to zero. The following equation shows the relationship between $\alpha_{h}$ and $\alpha$ at the takeoff position, assuming that $\alpha$ is equal to the fuselage angle of attack:

$$
\alpha_{h_{t o}}=\alpha_{t o}\left(1-\frac{d \varepsilon}{d \alpha}\right)+i_{h}-\varepsilon_{0}
$$

Equation 10 produces the maximum positive tail angle of attack, which should be below the tail stall angle. In contrast, the maximum positive elevator deflection should be examined during aircraft cruising with maximum speed, as well, to maintain the aircraft longitudinal trim.

The tail stall angle of attack at takeoff rotation is dependent on parameters such as: elevator deflection, elevator chord, and tail airfoil section. It can be expressed as:

$$
\alpha_{h_{s}}= \pm\left(\alpha_{h_{s: \delta e=0}}-\Delta \alpha_{h_{e}}\right)
$$

where:

$$
\begin{aligned}
& \alpha_{h_{s: \delta e=0}}=\text { typically around } 14 \text { degrees } \\
& \Delta \alpha_{h_{e}}=\text { the amount of reduction in the tail stall angle } \\
& \text { of attack }
\end{aligned}
$$

Table 2 [20] demonstrates the empirical values for $\Delta \alpha_{h_{e}}$ with respect to tail-to-elevator chord ratio and elevator deflection. Note that if the ratio $C_{e} / C_{h}$ is greater than 0.5 , it is recommended to use an all-moving tail instead.

Table 2: The reduction in stall angle at elevator deflection [20]

\begin{tabular}{|c|c|c|c|c|c|}
\hline \multirow{2}{*}{$\boldsymbol{\delta}_{\boldsymbol{e}}$ (deg) } & \multicolumn{5}{|c|}{ Tail-to-elevator chord ratio $C_{e} / C_{h}$} \\
\cline { 2 - 6 } & $\mathbf{0 . 1}$ & $\mathbf{0 . 2}$ & $\mathbf{0 . 3}$ & $\mathbf{0 . 4}$ & $\mathbf{0 . 5}$ \\
\hline $\mathbf{\pm 1 5}$ & 0.9 & 1.5 & 3.2 & 4.9 & 6.5 \\
\hline $\mathbf{\pm 2 0}$ & 1.2 & 2 & 4.2 & 6.5 & 8.7 \\
\hline $\mathbf{2 5}$ & 1.6 & 2.5 & 5.3 & 8.1 & 11 \\
\hline $\mathbf{\pm 3 0}$ & 1.9 & 3 & 6.4 & 9.7 & 13.1 \\
\hline
\end{tabular}


Finally, in the elevator sizing process, the lift coefficient of the horizontal tail is evaluated by employing lifting-line theory or CFD technique and should be compared with the desired lift coefficient. The parameter $\Delta \alpha_{0_{e}}$ is assessed in the next empirical equation and consecutively, employed to estimate the tail lift distribution and its coefficient:

$$
\Delta \alpha_{0_{e}} \approx-1.15 \times \frac{C_{e}}{C_{h}} \times \delta_{e}
$$

\section{ELEVATOR SIZING ALGORITHM}

For a conventional aircraft, the elevator sizing algorithm steps are:

1. Assigning and identifying the basic inputs of the algorithm which include: the ratio $b_{e} / b_{h}$, maximum up and down elevator deflection, and the takeoff angular acceleration from Table 1.

2. Applying Equations $1 \mathrm{a}$ and $2 \mathrm{a}$ to carry out the aircraft drag and the wing/fuselage lift, respectively.

3. Calculating the friction force, using Equation $1 e$. Then, determine the aircraft linear acceleration at rotation point, using Equation 1.

4. By considering the most forward aircraft center of gravity and using Equations $3 a-3 g$, determine all the required moments (i.e., $M_{W}, M_{D}, M_{T}, M_{L_{w f}}, M_{a c_{w f}}, M_{L_{h}}$ and $M_{a}$.

5. Using Equation 4 to evaluate the desired lift $L_{h}$.

6. Determine the desired lift coefficient $C_{L_{h}}$, using Equation 5.

7. Applying Equation 6 and its relatives to determine the elevator effectiveness $\tau_{e}$.

8. If the calculated $\tau_{e}$ is more than one, there is no elevator satisfying the prerequisite of takeoff rotation. Therefore, redesign the horizontal tail and/or landing gear and return to step 2 .

9. Calculating the corresponding chord ratio $\left(C_{e} / C_{h}\right)$, using Equation 7.

10. If the corresponding chord ratio is greater than 0.5 , it is recommended to use an all-moving tail.

11. Determine the deviation of the tail lift coefficient due to elevator deflection, using Equation 12. Then, applying lift-line theory or CFD to find the horizontal tail lift coefficient at the maximum negative elevator deflection. The MATLAB program in page 245 ref. [20] represents the general application of the lift-line theory and can be used here, by changing the input values as desired.

12. If the desired horizontal tail lift coefficient carried out from step 6 equal to the resultant coefficient for step 11, continue. If not equal, altering the elevator span and/or chord to adjust the resultant coefficient until getting the required value.
13. By considering the most forward and the most aft center of gravity of the aircraft with the helpfulness of Equations $8 \mathrm{a}$ and $8 \mathrm{~b}$, evaluate the elevator derivatives $C_{m_{\delta_{e}}}$ and $C_{L_{\delta_{e}}}$.

14. Calculating the required elevator deflection $\delta_{e}$ to achieve longitudinal trim, using Equation 8.

15. If the calculated maximum $\delta_{e}$ from the previous step is more than the assigned value in step 1, therefore, the elevator design does not satisfy the prerequisites of longitudinal trim. Resizing the horizontal tail and/ or landing gear and return to step 2 .

16. Determine the horizontal tail angle of attack, using Equation 10.

17. Evaluating the horizontal tail stall angle, using Equation 11. Refer to Table 2, to select a proper $\Delta \alpha_{h_{e}}$ value.

18. If the calculated angle of step 16 is greater than that of step 17, then the designed horizontal tail will stall. Resizing the elevator by decreasing the elevator chord and/or deflection. If the resizing is failed too, relocating or redesigning the landing gear, horizontal tail, or the center of gravity of the aircraft. Return to step 2.

\section{TESTING THE ALGORITHM}

The algorithm can be coded in a high level language and encapsulated in any preliminary aircraft design software to aid students and fresh engineers to enhance their understanding and analyzing of the elevator sizing process. The following example illustrates the application of this algorithm as an instructional use.

\section{Example}

For an 80 passenger conventional transport aircraft with the following data delivered from the conceptual phase:

$m_{\text {to }}=32000 \mathrm{~kg}, v_{\mathrm{s}}=59 \mathrm{~m} / \mathrm{s}, v_{c}=250 \mathrm{~m} / \mathrm{s}$ (at $9700 \mathrm{~m}$ ), $T_{\text {max }}^{\text {to }}=9.88 \mathrm{kN}, I_{f}=34.3 \mathrm{~m}, C_{d o}=0.023, C_{10}=0.38, S=66$ $\mathrm{m}^{2}, A R=9.3, \lambda=0.24, i_{w}=2 \mathrm{deg}, C_{l \alpha}=C_{\text {law }}=5.71 / \mathrm{rad}$, $S_{h}=11.9 \mathrm{~m}^{2}, b_{h}=7.7 \mathrm{~m}, \lambda_{h}=0.3, C_{\text {lah }}=4.31 / \mathrm{rad}, i_{h}=-1$ $\operatorname{deg}, C_{\text {lflap }}=0.43, C_{m \text { ac }}=-0.08$

Design a low-cost, easy-manufacturing elevator.

\section{Solution}

Step 1- The ratio $b_{e} / b_{h}$ was assigned a value of 1 . The maximum down elevator deflection is selected to be 25 deg. Based on Table 1, a $6 \mathrm{deg} / \mathrm{s}^{2}$ takeoff pitch angular acceleration is selected.

Step 2- The aircraft drag $=6678 \mathrm{~N}$ and the wing/fuselage lift force $=124803 \mathrm{~N}$.

Step 3- The friction force $=10022 \mathrm{~N}$, assuming $\mu=0.04$, and the linear acceleration $=2.35 \mathrm{~m} / \mathrm{s}^{2}$.

Step 4- The moment of the aircraft due to wing/fuselage lift $=-48548 \mathrm{Nm}$, the moment of weight $=-375199 \mathrm{Nm}$, the moment of drag $=13356 \mathrm{Nm}$, the moment of thrust $=-80755 \mathrm{Nm}$, the moment of lift $=93421 \mathrm{Nm}$, and the 
moment of acceleration $=143213 \mathrm{Nm}$. Note that all $\mathrm{x}$ and $z$ dimensions, required to calculate the moments, are delivered from the geometry section of the conceptual design phase.

Step 5- Applying Equation 4, the desired horizontal tail lift $=-24435 \mathrm{Nm}$.

Step 6- The desired horizontal tail lift coefficient $=-0.96$, using Equation 5.

Step 7- Employing Equations $6 \mathrm{a}$ and $6 \mathrm{~b}$ to find the downwash effect, $\varepsilon_{o}=0.061 \mathrm{rad}, d_{\varepsilon} / d_{\alpha}=0.418 \mathrm{deg} / \mathrm{deg}$ and $\alpha h=-3.304 \mathrm{deg}$. Hence, the elevator effectiveness $=$ 0.644 , using Equation 6.

Step 8- The effectiveness of the elevator is less than one, so, continue.

Step 9- The corresponding cord ratio $\left(C / C_{h}\right)=0.456$ from Figure 1 or using Equation 7.

Step 10- The ratio from step 9 is less than 0.5 , therefore, continue.

Step 11- Applying Equation 12 yields, $\Delta \alpha_{0_{e}}=13 \mathrm{deg}$. Figure 2 shows the distribution of the horizontal tail lift. The resultant lift coefficient $=-0.925$.

Step 12- Since, the deviation between the resultant coefficient and the required is too small, so, the elevator sizing is accepted.

Step 13- The elevator derivatives $C_{m_{\delta_{e}}}=-3.881 / \mathrm{rad}$, and $C_{L_{\delta_{e}}}=0.4791 / \mathrm{rad}$

Step 14-Applying Equation 8, $\delta_{e}=-0.263 \mathrm{deg}$.

Step 15- The resultant $\delta_{e}$ is less than the assigned value, in step 1.

Step 16- The horizontal tail angle of attack $=1.355 \mathrm{deg}$.

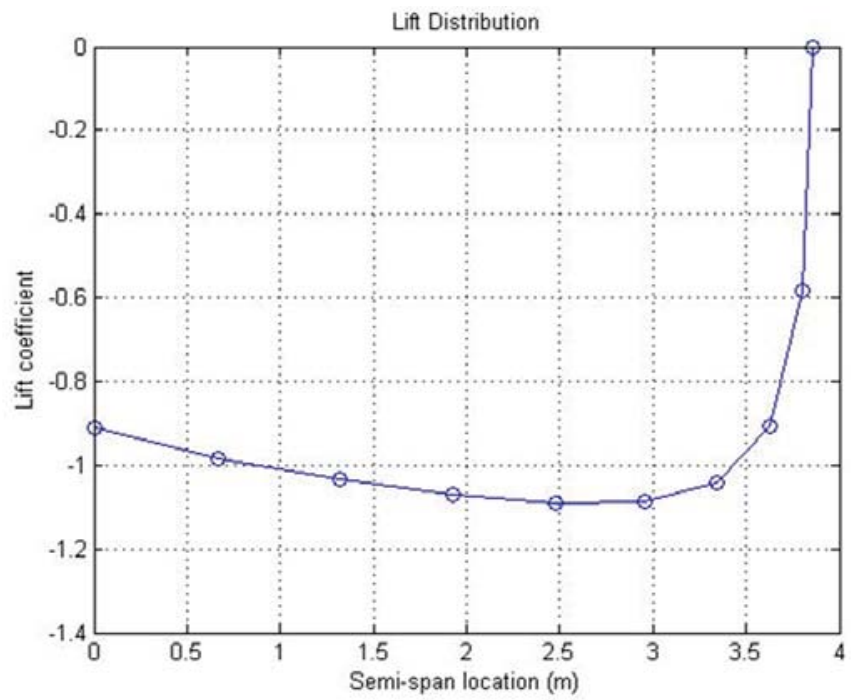

Figure 2: Tail lift distribution at -25 deg elevator deflection
Step 17- According to Table 2, the reduction in stall angle $=9.8 \mathrm{deg}$, and hence, the horizontal tail stall angle $=4.2$ deg.

Step 18- Since the difference between the horizontal tail angle of attack (1.355) and the stall angle (4.2) is greater than $2 \mathrm{deg}$, the elevator design is accepted.

The geometry of the designed elevator is:

$$
\begin{aligned}
& b_{e} b_{h}=1 \rightarrow b_{e}=b_{h}=7.7 \mathrm{~m}, C_{h}=S_{h} b_{h}=11.9 / 7.7=1.545 \mathrm{~m} \\
& C_{e} C_{h}=0.456 \rightarrow C_{e}=0.705 \mathrm{~m}, S_{e}=b_{e} C_{e}=5.426 \mathrm{~m}^{2}
\end{aligned}
$$

\section{CONCLUSIONS}

An instructive algorithm for the aircraft elevator sizing has been presented to be used in the preliminary aircraft design software. For fresh engineers and aeronautical students, this algorithm is useful to enhance their knowledge, understanding, and analyzing studies. The paper introduced the necessary formulae as a guide to size the elevator to achieve the longitudinal control and longitudinal trim requirements with a solved example, as an instructional use, step by step, to explain the application of the algorithm.

\section{REFERENCES}

1. R. Struett, "Empennage Sizing and Aircraft Stability using Matlab," California Polytechnic State University, San Luis Obispo, USA, 2012.

2. B. Etkin and L. Reid, Dynamics of Flight, Stability, and Control, USA: John Wiley \& Sons, Inc., 1996.

3. J. Roskam and S. Malaek, "Automated Aircraft Configuration Design and Analysis," SAE, no. paper 891072, pp. 271-288, April, 1989.

4. J. Roskam, Airplane Design, USA: published by author as an eight-volume set, 1985-2007.

5. D. Raymer, "RDS-Proffessional in action: Aircraft Design on a Personal Computer," SAE/AIAA, no. paper 5567, October, 1996.

6. D. Raymer, Aircraft design: A Conceptual Approach, USA: 4th edition, AIAA, 2006.

7. R. Kaenel, A. Rizzi, J. Oppelatrup, T. Geotzzendort-Grabowaki, M. Ghoreyshi, L.Cavagna and A. Berard, "CEASIOM: Simulating Stability and Control with CDF/CSM in Aircraft Conceptual Design," Alaska, USA, September, 2008.

8. F. Nicolosi and G. Paduano, "Development of a software for aircraft preliminary design and analysis (ADAS)," in 10th European Workshop on Aircraft Design Education, Napoli, Italy, May 24-27, 2011.

9. B. Chang, M. Bayram, H. Kwatny and C. Belcastro, "Flight path and altitude tracking control of an impaired nonlinear generic transport model(GTM) aircraft with elevator jam failures," in Control Applications (CCA), IEEE Conference (pp. 15-20), Buenos Aires, Argentina, 2016. 
10. X. Wang, S. Wang, Z. Yang and C. Zhang, "Active fault-tolerant control strategy of large civil aircraft under elevator failures," Chinese Journal of Aeronautics, vol. 28, no. 6, pp. 1658-1666, 2015.

11. A. Gaspari, F. Toffol, P. Mantegazza and A. Mannarino, "Optimal and robust design of a control surface actuation system within the GLAMOUR project," Aertecnica Missili \& Spazio, vol. 95, no. 4, 2016.

12. F. Oliviero, D. Zanetti and V. Cipolla, "Flight dynamics model for preliminary design of PrandtIPlane wing configuration with sizing of the control surfaces," Aerotechnica Missili \& Spazio, vol. 95, no. 4, pp. 201-210, 2016.

13. D. v. Ginneken, M. Voskuijl, M. v. Tooren and A. Frediam, "Automated Control Surface Design and Sizing for the Prandtl Plane," in 51st AIAA/ASME/ ASCE/AHS/ASC Structures, Structural Dynamics, and Materials Conference (AIAA 2010-3060), Orlando, Florida, 2010.
14. A. Emhemad, "On the Development of Computer Code for Aircraft Flight Dynamics Analysis," MSc thesis, Universiti Tun Hussien Onn Malaysia, 2014.

15. R. Nelson, Flight Stability and Automatic Control, USA: McGraw-Hill, 1989.

16. P. Barua, T. Sousa and D. Scholz, "Empennage Statistics and Sizing Methods for Dorsal Fins," Hamburg University of Applied Sciences, Hamburg, Germany, 2013.

17. I. Abbott and A. V. Doenhoff, Theory of Wing Sections, New York: Dover, 1959.

18. J. Roskam, Airplane Flight Dynamics and Automatic Flight Control, DAR Corporation, 2007.

19. MathWorks, "MATLAB," MathWorks, [Online]. Available: http://www.mathworks.co.uk/products/matlab/.

20. M. Sadraey, Aircraft Design: A Systems Engineering Approach, USA: John Wiley \& Sons, Ltd., 2013. 